Article

\title{
Order-stability in complex biosystems from the viewpoint of the theory of open quantum systems
}

\author{
Andrei Khrennikov ${ }^{1, *}$ and Noboru Watanabe ${ }^{2}$ \\ 1 International Center for Mathematical Modeling in Physics and Cognitive Sciences, Linnaeus University, \\ SE-351 95 Växjö, Sweden \\ 2 Department of Information Sciences, Tokyo University of Science \\ Noda City, Chiba 278-8510, Japan; watanabe@is.noda.tus.ac.jp \\ * Correspondence: Andrei.Khrennikov@lnu.se
}

\begin{abstract}
This paper is our attempt on the basis of physical theory to bring more clarification on the question "What is life?" formulated in the well-known book of Schrödinger in 1944. According to Schrödinger, the main distinguishing feature of biosystem's functioning is the ability to preserve its order structure or, in the mathematical terms, to prevent increasing of entropy. Since any biosystem is fundamentally open, it is natural to use open system's theory. However, Schrödinger's analysis shows that the classical theory is not able to adequately describe the order-stability in a biosystem. Schrödinger should also appeal to the ambiguous notion of negative entropy. We suggest to apply the quantum theory. As is well-known, behaviour of the quantum von Neumann entropy crucially differs from behaviour of the classical entropy. We consider a complex biosystem $S$ composed of many subsystems, say proteins, or cells, or neural networks in the brain, i.e., $S=\left(S_{i}\right)$. We study the following problem: if the composed system $S$ can preserve the "global order" in the situation of increase of local disorder and if $S$ can preserve its entropy while some of $S_{i}$ increase their entropies We show that within quantum information theory the answer is positive. The significant role plays entanglement of the subsystems states. In the absence of entanglement, increasing of local disorder generates disorder increasing in the compound system $S$ (as in the classical regime).
\end{abstract}

Keywords: biosystems; order-stability; classical versus quantum entropy; open quantum systems; quantum channel; entanglement

\section{Introduction}

This paper is motivated by Schrödinger's book [2] in that he considered one of the most fundamental and intriguing problems of modern science: "What is life?" This was the attempt to proceed towards clarification of this problem on the basis of quantum physics and thermodynamics.

Of course, from the purely biological viewpoint this attempt to resolve the basic problem of biology in the purely physical framework may be considered as very naive. Schr'odinger by himself pointed out the casualty of his approach. At the same the treatment of "What is life?" question in the purely physical framework can have its advantages; in particular, clarifying cleaning the biological details may enlighten a few basic issues related to this question.

\subsection{Order-stability as a distinguishing feature of biosystems}

Schrödinger tried to find analogies between physical and biological systems' functioning. And he emphasizes the amazing ability for order preservation as one of the basic features of functioning of biological systems. He compares this feature of latter with thermodynamics of physical systems governed by the Second Law of Thermodynamics: 
"What is the characteristic feature of life? When is a piece of matter said to be alive? It is alive when it goes on 'doing something', moving, exchanging material with its environment, and so forth, and that for a much longer period than we would expect of an inanimate piece of matter to 'keep going' under similar circumstances."

At the same time he stresses that, although any biosystem tries to minimize its coupling with surrounding environment, a completely isolated biosystem is dead. Thus, biosystems form a subclass of open systems that are weakly coupled with environments.

Entropy is the basic measure of disorder in physics and information theory. So, to be stable, a biosystem should be able to control entropy and prevent its essential increasing. (It is clear that the bio-entropy can fluctuate.) Schrödinger views the heuristic mechanism of entropy-stabilization through its emission from a system $S$ to the environment $\mathcal{E}$, i.e., through increasing of disorder in $\mathcal{E}$. Such system's behaviour doesn't match the laws of physics [2]: "When a system that is not alive is isolated or placed in a uniform environment, all motion usually comes to a standstill..." Schrödinger continues to speculate and suggests that $S$ absorbs the flow of "negative entropy" from $\mathcal{E}$. From the viewpoint of conventional physics, this notion is ambiguous and he points out that the mystery of life cannot be explained without discovery of new physical laws.

\subsection{Information biology and physics}

The book [2] stimulated creation of a new area of science which is nowadays is known as information biology by emphasizing that order stability or even its improvement for the alive-state cannot be modelled solely in terms of the energy and matter flows between a biosystem $S$ and the environment $\mathcal{E}$. Biosystems should be viewed as open systems interacting with the physical and information components of the surrounding environment. Since 1970s, the information's role was highlighted in biology, see, e.g., the well known paper of Johnson [3] characterizing information theory as a "general calculus for biology". As was pointed by Gatenby and Frieden [4], "it is clear that life without matter and energy is impossible, Johnson's manuscript emphasizes that life without information is likewise impossible. Since the article, remarkable progress has been made towards understanding the informational fundament for life..." This information reconstruction of biology was closely related to the similar process in physics, starting with Wheeler's "It from bit" [5] to the recent quantum information revolution. The latter led to information reconsideration of quantum foundations [6]-[12]. Therefore, quantum information and open quantum systems [13] can contribute to modelling of information interaction of the biosystem $S$ and the environment $\mathcal{E}$ (see monograph [14]).

\subsection{Quantum-like models}

This is a good place to make the remark on quantum and quantum-like modeling in biology. The first one is known as quantum biophysics and it describes the genuine quantum processes in biosystems. It operates on micro-scales, see, for example, the series of works [15]-[19] on modeling cognition from genuine quantum physical processes in the brain. . Schrödinger's book was the first step in this direction. (Maybe the global aim of quantum biophysics is too ambitious - to reduce biological functions, as say psychological functions, to quantum physical processes. The difference in scales, for space, time, temperature, is too big.) In the quantum-like modeling, a biosystem is characterized from the purely information processing viewpoint, i.e., its size and other scales, say of temperature, are not important. As was shown in numerous studies (mainly in cognition, psychology, decision making, but even microbiology) [20]-[61], in some contexts biosystems process information in accordance with the quantum laws. Thus, they can be considered as quantum-like (although not genuinely quantum). In this paper, we proceed in the quantum-like framework.

One may point to difference in the classical and quantum probabilistic descriptions [62-64] and question applicability of quantum probability calculus and, hence, quantum information theory to macroscopic biosystems. However, interrelation between these two descriptions is a complex problem of quantum foundations which we are not able to discuss in this paper (see, e.g., [65]-[67]). 


\subsection{Order-stability in a biosystem compounded of a few subsystems: quantum information approach}

In this paper, we want to model the order stability inside of a complex biosystem $S$ that is composed of a few subsystems $S_{i}, i=1,2 \ldots, N$. We study the following problem of big complexity:

Can a composed system $S=\left(S_{i}\right)$ preserve the "global order" in itself, in spite of increase of local disorder (i.e., in its subsystems)?

In the mathematical framework, this question is formulated as follows:

Can $S=\left(S_{i}\right)$ preserve its entropy while some of its subsystems $S_{i}$ (or even all) increase their entropies?

We show that within quantum information theory the answer is positive.

The key point is that in quantum theory the state of a compound system is not reduced to the states of its subsystems. The entropy balance in $S$ is not based on summation of the entropies generated by subsystems. Here, the significant role is played by entanglement, nonclassical correlations between the states of subsystems $S_{i}$ of $S$. In the absence of entanglement, entropy behaves classically: the entropy of $S$ equals the sum of entropies of $S_{i}$.

We explore the following feature of quantum channels (dynamical maps describing the state evolution): they can transfer non-entangled states into entangled. By using this feature we present the scheme of the concrete quantum channels construction preserving the global entropy and increasing all local entropies. The construction is technically quite complicated. We restrict considerations to the case of two subsystems. We start with qubit state spaces of the subsystems and then consider the general case of $\mathrm{N}$-dimensional state spaces. Our construction is explicitly based on representation of channels through orthonormal bases in the subsystems state spaces.

This construction of desired quantum channels is restricted to the unitary evolution of the $S$-state. Generalization to non-unitary channels and complex systems with a large number of subsystems will be presented in future publication.

Finally, we remark that our framework and the result on entropy-stability can be applied not only to biosystemns compounded of subsystems, say organs compounded of cells or organisms compounded of organs, but also to social science and management (see the paper of Lawless [58] who also mentioned coupling with Schrödinger's book [2]).

\section{A few words about the quantum formalism}

Denote by $\mathcal{H}$ a complex Hilbert space endowed with the scalar product $\langle\cdot \mid \cdot\rangle$. For simplicity, we assume that it is finite dimensional. The space of density operators is denoted by $\mathfrak{S}(\mathcal{H})$ The space of all linear operators in $\mathcal{H}$ is denoted by the symbol $\mathcal{L}(\mathcal{H})$. In turn, this is the complex Hilbert space with the scalar product, $\langle A \mid B\rangle=\operatorname{Tr} A^{\star} B$. We shall also consider linear operators acting in $\mathcal{L}(\mathcal{H})$. They are called superoperators.

A pure quantum state is represented by a vector $|\psi\rangle \in \mathcal{H}$ that is normalized by 1 , i.e., $\langle\psi \mid \psi\rangle=1$. It can be represented as the density operator $\rho_{\psi}=|\psi\rangle\langle\psi|$, this is the orthogonal projector on the vector $|\psi\rangle$. States which are not pure are called mixed.

\subsection{Von Neumann entropy}

The von Neumann entropy is defined as

$$
\mathcal{S}(\rho)=- \text { Træ ln } æ,
$$

where $\rho$ is a density operator.

There exists an orthonormal basis $\left(e_{j}\right)$ consisting of eigenvectors of $\rho$, i.e., $\rho e_{j}=p_{j} e_{j}$ (where $p_{j} \geq 0$ and $\left.\sum_{j} p_{j}=1\right)$. In this basis, the matrix of the operator $\rho \ln \rho$ has the form $\operatorname{diag}\left(\mathrm{p}_{\mathrm{j}} \ln \mathrm{p}_{\mathrm{j}} ;\right)$ hence

$$
\mathcal{S}(\rho)=-\sum_{j} p_{j} \ln p_{j} .
$$


However, the von Neumann entropy has the Shannon form only w.r.t. this special basis.

We present three basic properties of the von Neumann entropy.

1. $\mathcal{S}(\rho)=0$ if and only if $\rho$ is a pure quantum state, i.e., $\rho=|\psi\rangle\langle\psi|$.

2. For a unitary operator $U, \mathcal{S}\left(U \rho U^{\star}\right)=\mathcal{S}(\rho)$.

3. The maximum of entropy is approached on the state $\rho_{\text {disorder }}=I / N$ and $\mathcal{S}\left(\rho_{\text {disorder }}\right)=\ln N$, where $N$ is the dimension of the state space.

It is natural to call $\rho_{\text {disorder }}=I / N$ the state of maximal disorder.

For an isolated quantum system, dynamics is represented by unitary evolution operator $U(t)$. Thus, in contrast to the classical case, the entropy of an isolated quantum system is not changed with time. Hence, system's order structure is preserved; disorder inside it is not increased with time. Behavior of the quantum entropy significantly differs from behavior of the classical entropy. For the latter, the Second Law of Thermodynamics implies entropy's increase with time and, hence, destruction of order in the system.

\subsection{States of a compound system and its subsystems, entanglement}

Let $S=\left(S_{1}, S_{2}\right)$ be a compound system represented in Hilbert space $\mathcal{H}_{1} \otimes \mathcal{H}_{2}$ and let $\rho \in$ $\mathfrak{S}\left(\mathcal{H}_{1} \otimes \mathcal{H}_{2}\right)$. The states of its subsystems are calculated as the partial traces of $\rho$ :

$$
\rho_{1}=\operatorname{Tr}_{\mathrm{H}_{2}} \rho, \rho_{2}=\operatorname{Tr}_{\mathrm{H}_{1}} \rho,
$$

$\rho_{i} \in \mathfrak{S}\left(\mathcal{H}_{i}\right)$.

Consider now a pure state of $S$ that is factorisable w.r.t. the tensor product structure, i.e., $|\Psi\rangle=$ $\left|\psi_{1}\right\rangle \otimes\left|\psi_{2}\right\rangle$. States which are not represented in this form are called entangled. Entangled states plays the crucial role in quantum information theory. In particular, this is the most important resource of quantum computations. They represent the correlations between subsystems of a quantum system. These correlations are nonclassical, in the sense that they cannot be adequately described by the classical probability, the Kolmogorov measure-theoretic axiomatics [62].

The definition of entanglement can be generalized to mixed states. A state $\rho \in \mathfrak{S}\left(\mathcal{H}_{1} \otimes \mathcal{H}_{2}\right)$ is called separable if it can be represented in the form:

$$
\rho=\sum_{k m} c_{k m} \rho_{1}^{(k)} \otimes \rho_{2}^{(m)}
$$

where $\rho_{i}^{(j)} \in \mathfrak{S}\left(\mathcal{H}_{i}\right), i=1,2$. A compound state that cannot be represented in this form is called entangled. However, in this paper we shall consider only entanglement of pure states.

Generally the entropies of the compound system $S$ and its subsystems $S_{i}$ are constrained by the inequality:

$$
\left|\mathcal{S}\left(\rho_{2}\right)-\mathcal{S}\left(\rho_{1}\right)\right| \leq \mathcal{S}(\rho) \leq \mathcal{S}\left(\rho_{1}\right)+\mathcal{S}\left(\rho_{2}\right) .
$$

If the state $\rho$ is separable, then

$$
\mathcal{S}(\rho)=\mathcal{S}\left(\rho_{1}\right)+\mathcal{S}\left(\rho_{2}\right) .
$$

This is the classical situation and the local entropy's increase, in any subsystem, implies the global entropy's increase on the same amount. On the other hand, if the state $\rho$ is entangled, then equality (6) is violated; general inequality (5) permits consistent increase of the entropies of the both subsystems without increase of the entropy of the compound system.

Consider now the pure state $\rho_{\Psi}=|\Psi\rangle\langle\Psi|$, where $|\Psi\rangle \in \mathcal{H}_{1} \otimes \mathcal{H}_{2}$. The states of the systems $S_{i}$ are pure if and only if $|\Psi\rangle$ is separable. Thus, for an entangled state $\rho_{\Psi}$, the states $\rho_{i}$ are always mixed states.

This fact is important for our further study. It implies that, for an entangled pure state, the entropies of subsystem's states $\mathcal{S}\left(\rho_{i}\right)>0$, because $\rho_{i}$ is not pure. 


\subsection{Open quantum system dynamics, quantum channels}

Consider evolution of the state of the compound system $S=\left(S_{1}, S_{2}\right), \rho(t)=\Lambda_{t} \rho_{0}$ and the corresponding evolution of the states of $S_{i}$,

$$
\rho_{1}(t)=\operatorname{Tr}_{H_{2}} \Lambda_{t} \rho_{0}, \rho_{2}(t)=\operatorname{Tr}_{H_{1}} \Lambda_{t} \rho_{0}
$$

In the framework of open quantum systems theory, for each $t$, state's evolution of $S$ is represented by a quantum channel - trace-preserving completely positive map (superoperator) acting in the space $\mathcal{L}\left(\mathcal{H}_{1} \otimes \mathcal{H}_{2}\right)$. We remark that quantum channel $\Lambda_{t}$ also generate quantum channels for subsystems' states; they are given by superoperators

$$
\Lambda_{1 t} \rho_{1}=\operatorname{Tr}_{H_{2}} \Lambda_{t} \rho_{1} \otimes I, \Lambda_{2 t} \rho_{2}=\operatorname{Tr}_{H_{1}} \Lambda_{t} I \otimes \rho_{2}
$$

Each subsystem $S_{i}$ of the compund system $S$ can be considered as open quantum system. In the case of the isolated system $S, S_{2}$ plays the role of the environment of $S_{1}$ and vice verse. If $S$ is not isolated, the environment of $S_{1}$ includes $S_{2}$ and the environment of $\mathcal{E}_{S}$ of $S$.

\section{Formulation of the problem}

We are interested in the condition of order-stability in the compound system $S$ in the situation of disorder-increasing in its subsystems $S_{i}$, i.e., in the possibility that, for some $t>0$,

$$
\mathcal{S}\left(\rho_{i}(t)\right)>\mathcal{S}\left(\rho_{0 i}\right), i=1,2 \text {, but } \mathcal{S}(\rho(t))=\mathcal{S}\left(\rho_{0}\right) \text {. }
$$

If the initial state of the compound system $S$ is factorizable (non-entangled), i.e., $\rho_{0}=\rho_{01} \otimes \rho_{02}$, then condition (9) can be rewritten as

$$
\mathcal{S}\left(\Lambda_{i t} \rho_{0 i}\right)>\mathcal{S}\left(\rho_{0 i}\right), i=1,2 \text {, but } \mathcal{S}\left(\Lambda_{t} \rho_{0}\right)=\mathcal{S}\left(\rho_{0}\right)
$$

where subsystems' quantum channels are defined by (8). In our model, we shall consider precisely this case: non-entangled initial state $\rho_{0}$ of the compound system.

The simplest model of such behavior is based on the unitary evolution of $S$, i.e., one parameteric group of unitary operators $U_{t}: \mathcal{H}_{1} \otimes \mathcal{H}_{2} \rightarrow H_{1} \otimes \mathcal{H}_{2}$. In this case, $\Lambda_{t} \rho_{0}=U_{t} \rho_{0} U_{t}^{\star}$. Such dynamics transfers pure states into pure states. Hence, $\left.\mathcal{S}\left(\Lambda_{t} \rho_{0}\right)=\mathcal{S}\left(\rho_{0}\right)\right)=0$. If $\rho_{0}$ corresponds to a separable pure state, then $\mathcal{S}\left(\rho_{0 i}\right)=0, i=1,2$, as well.

If the quantum channel $\Lambda_{t}$ transfers a separable state into an entangled state, then $\rho_{i}(t), i=1,2$, are mixed states and, hence, they have positive entropy. Thus, our desire is to construct a unitary evolution operator that can transfer separable states into entangled states. It is well-known that such operators exist and they are widely used in quantum computations. We shall construct the concrete operators for state spaces of an arbitrary (finite) dimension. These operatores are explicitly expressed through orthonormal bases in Hilbert spaces $\mathcal{H}_{i}$ and entropy increase can be exactly calculated.

\section{Complex systems}

A biosystem $S$ is typically composed of a large number of subsystems $S_{i}, i=1,2, \ldots, M$ (say genes, proteins, cells, organs, neural networks). Let subsystem $S_{i}$ is represented in Hilbert space $\mathcal{H}_{i}$. The compound system $S$ is represented in tensor product $\mathcal{H}=\otimes_{i=1} \mathcal{H}_{i}$. For quantum state $\rho \in \mathfrak{S}(\mathcal{H})$, states of the subsystems are given by partial traces $\rho_{j}=\operatorname{Tr}_{\otimes_{i \neq j} \mathcal{H}_{i}} \rho$. Let $\Lambda_{t}$ be a quantum channel describing the dynamics of the compound state, $\rho(t)=\Lambda_{t} \rho_{0}$; then the states of subsystems evolves as $\rho_{j}(t)=\operatorname{Tr}_{\otimes_{i \neq j} \mathcal{H}_{i}} \Lambda_{t} \rho_{0}$. For the fixed subsystem $S_{j}$, the system $S_{j}^{\prime}=\left(S_{i}\right)_{i \neq j}$ plays the role of its environment (in the case of isolated $S$ ). We are interested in generalization of conditions (9), (10) for $i=1,2, \ldots, M$. However, even in the case $M=2$ considered in this paper calculations are long. We do not want to overshadow the main idea of compound-stability by even longer calculations. Although 
calculations for an arbitrary $M$ are more complicated, but it is clear that desired quantum channles can be constructed, especially for spaces of the dimension $\operatorname{dim} \mathcal{H}=2^{\mathrm{M}}$, for $M$ qubit spaces.

\section{Quantum channel preserving compound system's entropy, in spite increasing of subsystems' entropies}

Our constructions of the desired quantum channel for subsystem's state spaces of dimensions $N=2$ and $N>2$ are different. In the latter case, the expressions for the von Neumann entropies of the subsystems $S_{i}, i=1,2$, contain the factor $\log (N-2)$. Therefore, we consider these cases separately.

\subsection{Two subsystems with qubit state spaces}

Let $\mathcal{H}_{1}$ and $\mathcal{H}_{2}$ be $\mathbb{C}^{2}$ and $\left\{\left|x_{0}^{(i)}\right\rangle,\left|x_{1}^{(i)}\right\rangle\right\}$ be a CONS in $\mathcal{H}_{i}(i=1,2)$. We define a completely positive channel $\Lambda^{*}$ from $\mathfrak{S}\left(\mathcal{H}_{1} \otimes \mathcal{H}_{2}\right)$ to $\mathfrak{S}\left(\mathcal{H}_{1} \otimes \mathcal{H}_{2}\right)$ by

$$
\Lambda^{*}(\bullet) \equiv V(\bullet) V^{*}
$$

where $V$ is a linear map from $\mathcal{H}_{1} \otimes \mathcal{H}_{2}$ to $\mathcal{H}_{1} \otimes \mathcal{H}_{2}$ given by

$$
\begin{aligned}
V \equiv & \sqrt{\frac{1}{2}}\left(\left|x_{0}^{(1)}\right\rangle \otimes\left|x_{0}^{(2)}\right\rangle+\left|x_{1}^{(1)}\right\rangle \otimes\left|x_{1}^{(2)}\right\rangle\right)\left\langle x_{0}^{(1)}\right| \otimes\left\langle x_{0}^{(2)}\right| \\
& +\sqrt{\frac{1}{2}}\left(\left|x_{0}^{(1)}\right\rangle \otimes\left|x_{0}^{(2)}\right\rangle-\left|x_{1}^{(1)}\right\rangle \otimes\left|x_{1}^{(2)}\right\rangle\right)\left\langle x_{1}^{(1)}\right| \otimes\left\langle x_{1}^{(2)}\right| \\
& +\sqrt{\frac{1}{2}}\left(\left|x_{0}^{(1)}\right\rangle \otimes\left|x_{1}^{(2)}\right\rangle+\left|x_{1}^{(1)}\right\rangle \otimes\left|x_{0}^{(2)}\right\rangle\right)\left\langle x_{0}^{(1)}\right| \otimes\left\langle x_{1}^{(2)}\right| \\
& +\sqrt{\frac{1}{2}}\left(\left|x_{0}^{(1)}\right\rangle \otimes\left|x_{1}^{(2)}\right\rangle-\left|x_{1}^{(1)}\right\rangle \otimes\left|x_{0}^{(2)}\right\rangle\right)\left\langle x_{1}^{(1)}\right| \otimes\left\langle x_{0}^{(2)}\right|
\end{aligned}
$$

We remark that the operator $V$ is unitary (see Lemma 1 in Appendix A). Thus this channel is noiseless - it is given by the unitary dynamics.

Let $\Phi$ be an initial compound state on $\mathcal{H}_{1} \otimes \mathcal{H}_{2}$ denoted by

$$
\Phi=\left(\left|x_{0}^{(1)}\right\rangle \otimes\left|x_{0}^{(2)}\right\rangle\right)\left(\left\langle x_{0}^{(1)}\right| \otimes\left\langle x_{0}^{(2)}\right|\right)
$$

One has the von Neumann entropy of $\Phi$ such as

$$
S(\Phi)=0
$$

The two marginal states of $\Phi$ are

$$
\rho_{1}=\left|x_{0}^{(1)}\right\rangle\left\langle x_{0}^{(1)}\left|, \rho_{2}=\right| x_{0}^{(2)}\right\rangle\left\langle x_{0}^{(2)}\right|
$$

The von Neumann entropy of two marginal states $\rho_{1}$ and $\rho_{2}$ are

$$
S\left(\rho_{1}\right)=0, S\left(\rho_{2}\right)=0
$$

The final compound state $\Lambda^{*}(\Phi)$ transmitted through the CP channel is

$$
\Lambda^{*}(\Phi)=\sqrt{\frac{1}{2}}\left(\left|x_{0}^{(1)}\right\rangle \otimes\left|x_{0}^{(2)}\right\rangle+\left|x_{1}^{(1)}\right\rangle \otimes\left|x_{1}^{(2)}\right\rangle\right) \sqrt{\frac{1}{2}}\left(\left\langle x_{0}^{(1)}\right| \otimes\left\langle x_{0}^{(2)}\right|+\left\langle x_{1}^{(1)}\right| \otimes\left\langle x_{1}^{(2)}\right|\right)
$$

We emphasize that this is an entangled state. 
We also have the von Neumann entropy of $\Lambda^{*}(\Phi)$ is

$$
S\left(\Lambda^{*}(\Phi)\right)=0=S(\Phi)
$$

The two marginal states of $\Lambda^{*}(\Phi)$ are

$$
\Lambda_{1}^{*} \rho_{1}=\frac{1}{2}\left|x_{0}^{(1)}\right\rangle\left\langle x_{0}^{(1)}\left|+\frac{1}{2}\right| x_{1}^{(1)}\right\rangle\left\langle x_{1}^{(1)}\left|, \Lambda_{2}^{*} \rho_{2}=\frac{1}{2}\right| x_{0}^{(2)}\right\rangle\left\langle x_{0}^{(2)}\left|+\frac{1}{2}\right| x_{1}^{(2)}\right\rangle\left\langle x_{1}^{(2)}\right|
$$

The von Neumann entropy of two marginal states $\Lambda_{1}^{*} \rho_{1}$ and $\Lambda_{2}^{*} \rho_{2}$ are

$$
\begin{aligned}
& S\left(\Lambda_{1}^{*} \rho_{1}\right)=\log 2>S\left(\rho_{1}\right) \\
& S\left(\Lambda_{2}^{*} \rho_{2}\right)=\log 2>S\left(\rho_{2}\right)
\end{aligned}
$$

\subsection{Two subsystems with $N$-dimensional state spaces}

We expand the above setting to $N \times N$ compound systems $(N \geq 3)$.

Let $\mathcal{H}_{1}$ and $\mathcal{H}_{2}$ be $\mathbb{C}^{N}$ and $\left\{\left|x_{k}^{(i)}\right\rangle\right\}_{k=0}^{N-1}$ be a CONS in $\mathcal{H}_{i}(i=1,2)$. We define a completely positive channel $\Lambda^{*}$ from $\mathfrak{S}\left(\mathcal{H}_{1} \otimes \mathcal{H}_{2}\right)$ to $\mathfrak{S}\left(\mathcal{H}_{1} \otimes \mathcal{H}_{2}\right)$ by

$$
\Lambda^{*}(\bullet) \equiv V(\bullet) V^{*}
$$

where $V$ is a linear map from $\mathcal{H}_{1} \otimes \mathcal{H}_{2}$ to $\mathcal{H}_{1} \otimes \mathcal{H}_{2}$ given by

$$
V=\sum_{k, \ell=0}^{N-1}\left|\varphi_{k, \ell}\right\rangle\left\langle x_{k}^{(1)}\right| \otimes\left\langle x_{\ell}^{(2)}\right|,
$$

where

$$
\begin{aligned}
\left|\varphi_{k, \ell}\right\rangle & =\frac{2}{N} \sum_{j=0}^{N-1} \alpha_{k, \ell, j}\left|x_{j}^{(1)}\right\rangle \otimes\left|x_{j+k \bmod \mathrm{N}}^{(2)}\right\rangle \\
\alpha_{k, \ell, j} & =\left\{\begin{array}{cc}
-\frac{N-2}{2} & (j=\ell) \\
1 & (j \neq \ell)
\end{array} \quad(k=0,1,2, \cdots, N-1)\right.
\end{aligned}
$$




$$
\begin{aligned}
& V=\frac{2}{N}\left(\left(-\frac{N-2}{2}\right)\left|x_{0}^{(1)}\right\rangle \otimes\left|x_{0}^{(2)}\right\rangle+\left|x_{1}^{(1)}\right\rangle \otimes\left|x_{1}^{(2)}\right\rangle+\cdots+\left|x_{N-1}^{(1)}\right\rangle \otimes\left|x_{N-1}^{(2)}\right\rangle\right)\left\langle x_{0}^{(1)}\right| \otimes\left\langle x_{0}^{(2)}\right| \\
& +\frac{2}{N}\left(\left|x_{0}^{(1)}\right\rangle \otimes\left|x_{0}^{(2)}\right\rangle+\left(-\frac{N-2}{2}\right)\left|x_{1}^{(1)}\right\rangle \otimes\left|x_{1}^{(2)}\right\rangle+\cdots+\left|x_{N-1}^{(1)}\right\rangle \otimes\left|x_{N-1}^{(2)}\right\rangle\right)\left\langle x_{0}^{(1)}\right| \otimes\left\langle x_{1}^{(2)}\right| \\
& +\cdots+ \\
& +\frac{2}{N}\left(\left|x_{0}^{(1)}\right\rangle \otimes\left|x_{0}^{(2)}\right\rangle+\left|x_{1}^{(1)}\right\rangle \otimes\left|x_{1}^{(2)}\right\rangle+\cdots+\left(-\frac{N-2}{2}\right)\left|x_{N-1}^{(1)}\right\rangle \otimes\left|x_{N-1}^{(2)}\right\rangle\right)\left\langle x_{0}^{(1)}\right| \otimes\left\langle x_{N-1}^{(2)}\right| \\
& +\frac{2}{N}\left(\left(-\frac{N-2}{2}\right)\left|x_{0}^{(1)}\right\rangle \otimes\left|x_{1}^{(2)}\right\rangle+\left|x_{1}^{(1)}\right\rangle \otimes\left|x_{2}^{(2)}\right\rangle+\cdots+\left|x_{N-1}^{(1)}\right\rangle \otimes\left|x_{0}^{(2)}\right\rangle\right)\left\langle x_{1}^{(1)}\right| \otimes\left\langle x_{0}^{(2)}\right| \\
& +\frac{2}{N}\left(\left|x_{0}^{(1)}\right\rangle \otimes\left|x_{1}^{(2)}\right\rangle+\left(-\frac{N-2}{2}\right)\left|x_{1}^{(1)}\right\rangle \otimes\left|x_{2}^{(2)}\right\rangle+\cdots+\left|x_{N-1}^{(1)}\right\rangle \otimes\left|x_{0}^{(2)}\right\rangle\right)\left\langle x_{1}^{(1)}\right| \otimes\left\langle x_{1}^{(2)}\right| \\
& +\cdots+ \\
& +\frac{2}{N}\left(\left|x_{0}^{(1)}\right\rangle \otimes\left|x_{1}^{(2)}\right\rangle+\left|x_{1}^{(1)}\right\rangle \otimes\left|x_{2}^{(2)}\right\rangle+\cdots+\left(-\frac{N-2}{2}\right)\left|x_{N-1}^{(1)}\right\rangle \otimes\left|x_{0}^{(2)}\right\rangle\right)\left\langle x_{1}^{(1)}\right| \otimes\left\langle x_{N-1}^{(2)}\right| \\
& +\frac{2}{N}\left(\left(-\frac{N-2}{2}\right)\left|x_{0}^{(1)}\right\rangle \otimes\left|x_{2}^{(2)}\right\rangle+\left|x_{1}^{(1)}\right\rangle \otimes\left|x_{3}^{(2)}\right\rangle+\cdots+\left|x_{N-1}^{(1)}\right\rangle \otimes\left|x_{1}^{(2)}\right\rangle\right)\left\langle x_{2}^{(1)}\right| \otimes\left\langle x_{0}^{(2)}\right| \\
& +\frac{2}{N}\left(\left|x_{0}^{(1)}\right\rangle \otimes\left|x_{2}^{(2)}\right\rangle+\left(-\frac{N-2}{2}\right)\left|x_{1}^{(1)}\right\rangle \otimes\left|x_{3}^{(2)}\right\rangle+\cdots+\left|x_{N-1}^{(1)}\right\rangle \otimes\left|x_{1}^{(2)}\right\rangle\right)\left\langle x_{2}^{(1)}\right| \otimes\left\langle x_{1}^{(2)}\right| \\
& +\cdots+ \\
& +\frac{2}{N}\left(\left|x_{0}^{(1)}\right\rangle \otimes\left|x_{2}^{(2)}\right\rangle+\left|x_{1}^{(1)}\right\rangle \otimes\left|x_{3}^{(2)}\right\rangle+\cdots+\left(-\frac{N-2}{2}\right)\left|x_{N-1}^{(1)}\right\rangle \otimes\left|x_{1}^{(2)}\right\rangle\right)\left\langle x_{2}^{(1)}\right| \otimes\left\langle x_{N-1}^{(2)}\right| \\
& +\cdots \cdots+ \\
& +\frac{2}{N}\left(\left(-\frac{N-2}{2}\right)\left|x_{0}^{(1)}\right\rangle \otimes\left|x_{N-1}^{(2)}\right\rangle+\left|x_{1}^{(1)}\right\rangle \otimes\left|x_{0}^{(2)}\right\rangle+\cdots+\left|x_{N-1}^{(1)}\right\rangle \otimes\left|x_{N-2}^{(2)}\right\rangle\right)\left\langle x_{N-1}^{(1)}\right| \otimes\left\langle x_{0}^{(2)}\right| \\
& +\frac{2}{N}\left(\left|x_{0}^{(1)}\right\rangle \otimes\left|x_{N-1}^{(2)}\right\rangle+\left(-\frac{N-2}{2}\right)\left|x_{1}^{(1)}\right\rangle \otimes\left|x_{0}^{(2)}\right\rangle+\cdots+\left|x_{N-1}^{(1)}\right\rangle \otimes\left|x_{N-2}^{(2)}\right\rangle\right)\left\langle x_{N-1}^{(1)}\right| \otimes\left\langle x_{1}^{(2)}\right| \\
& +\cdots+ \\
& +\frac{2}{N}\left(\left|x_{0}^{(1)}\right\rangle \otimes\left|x_{N-1}^{(2)}\right\rangle+\left|x_{1}^{(1)}\right\rangle \otimes\left|x_{0}^{(2)}\right\rangle+\cdots+\left(-\frac{N-2}{2}\right)\left|x_{N-1}^{(1)}\right\rangle \otimes\left|x_{N-2}^{(2)}\right\rangle\right)\left\langle x_{N-1}^{(1)}\right| \otimes\left\langle x_{N-1}^{(2)}\right|
\end{aligned}
$$

The unitarity of the operator $V$ is proved in Appendix B. Hence, this channel is noiseless.

Let $\Phi$ be an initial compound state on $\mathcal{H}_{1} \otimes \mathcal{H}_{2}$ denoted by

$$
\Phi=\left(\left|x_{0}^{(1)}\right\rangle \otimes\left|x_{0}^{(2)}\right\rangle\right)\left(\left\langle x_{0}^{(1)}\right| \otimes\left\langle x_{0}^{(2)}\right|\right)
$$

One has the von Neumann entropy of $\Phi$ such as

$$
S(\Phi)=0
$$

The two marginal states of $\Phi$ are

$$
\rho_{1}=\left|x_{0}^{(1)}\right\rangle\left\langle x_{0}^{(1)}\left|, \rho_{2}=\right| x_{0}^{(2)}\right\rangle\left\langle x_{0}^{(2)}\right|
$$

The von Neumann entropy of two marginal states $\rho_{1}$ and $\rho_{2}$ are

$$
S\left(\rho_{1}\right)=0, S\left(\rho_{2}\right)=0
$$


The final compound state $\Lambda^{*}(\Phi)$ transmitted through the CP channel is

$$
\begin{aligned}
\Lambda^{*}(\Phi)= & \frac{2}{N}\left(\left(-\frac{N-2}{2}\right)\left|x_{0}^{(1)}\right\rangle \otimes\left|x_{0}^{(2)}\right\rangle+\sum_{k=1}^{N-1}\left|x_{k}^{(1)}\right\rangle \otimes\left|x_{k}^{(2)}\right\rangle\right) \\
& \frac{2}{N}\left(\left(-\frac{N-2}{2}\right)\left\langle x_{0}^{(1)}\right| \otimes\left\langle x_{0}^{(2)}\right|+\sum_{k=1}^{N-1}\left\langle x_{k}^{(1)}\right| \otimes\left\langle x_{k}^{(2)}\right|\right)
\end{aligned}
$$

We also have the von Neumann entropy of $\Lambda^{*}(\Phi)$ is

$$
S\left(\Lambda^{*}(\Phi)\right)=0=S(\Phi)
$$

The two marginal states of $\Lambda^{*}(\Phi)$ are

$$
\begin{aligned}
& \Lambda_{1}^{*} \rho_{1}=\frac{(N-2)^{2}}{N^{2}}\left|x_{0}^{(1)}\right\rangle\left\langle x_{0}^{(1)}\left|+\frac{4}{N^{2}} \sum_{k=1}^{N-1}\right| x_{k}^{(1)}\right\rangle\left\langle x_{k}^{(1)}\right|, \\
& \Lambda_{2}^{*} \rho_{2}=\frac{(N-2)^{2}}{N^{2}}\left|x_{0}^{(2)}\right\rangle\left\langle x_{0}^{(2)}\left|+\frac{4}{N^{2}} \sum_{k=1}^{N-1}\right| x_{k}^{(2)}\right\rangle\left\langle x_{k}^{(2)}\right|
\end{aligned}
$$

The von Neumann entropy of two marginal states $\Lambda_{1}^{*} \rho_{1}$ and $\Lambda_{2}^{*} \rho_{2}$ are

$$
\begin{aligned}
& S\left(\Lambda_{1}^{*} \rho_{1}\right)=2 \log N-\frac{2(N-1)^{2}}{N^{2}} \log (N-2)-\frac{8(N-1)}{N^{2}} \log 2>S\left(\rho_{1}\right) \\
& S\left(\Lambda_{2}^{*} \rho_{2}\right)=2 \log N-\frac{2(N-1)^{2}}{N^{2}} \log (N-2)-\frac{8(N-1)}{N^{2}} \log 2>S\left(\rho_{2}\right)
\end{aligned}
$$

Consider the above general formulas for the case $N=3$. Let $\Phi$ be an initial compound state on $\mathcal{H}_{1} \otimes \mathcal{H}_{2}$ denoted by

$$
\Phi=\left(\left|x_{0}^{(1)}\right\rangle \otimes\left|x_{0}^{(2)}\right\rangle\right)\left(\left\langle x_{0}^{(1)}\right| \otimes\left\langle x_{0}^{(2)}\right|\right)
$$

One has the von Neumann entropy of $\Phi$ such as

$$
S(\Phi)=0
$$

The two marginal states of $\Phi$ are

$$
\rho_{1}=\left|x_{0}^{(1)}\right\rangle\left\langle x_{0}^{(1)}\left|, \rho_{2}=\right| x_{0}^{(2)}\right\rangle\left\langle x_{0}^{(2)}\right|
$$

The von Neumann entropy of two marginal states $\rho_{1}$ and $\rho_{2}$ are

$$
S\left(\rho_{1}\right)=0, S\left(\rho_{2}\right)=0
$$

The final compound state $\Lambda^{*}(\Phi)$ transmitted through the CP channel is

$$
\begin{aligned}
\Lambda^{*}(\Phi)= & \frac{2}{3}\left(\left|x_{0}^{(1)}\right\rangle \otimes\left|x_{0}^{(2)}\right\rangle+\left|x_{1}^{(1)}\right\rangle \otimes\left|x_{1}^{(2)}\right\rangle-\frac{1}{2}\left|x_{2}^{(1)}\right\rangle \otimes\left|x_{2}^{(2)}\right\rangle\right) \\
& \frac{2}{3}\left(\left\langle x_{0}^{(1)}\right| \otimes\left\langle x_{0}^{(2)}\right|+\left\langle x_{1}^{(1)}\right| \otimes\left\langle x_{1}^{(2)}\right|-\frac{1}{2}\left\langle x_{2}^{(1)}\right| \otimes\left\langle x_{2}^{(2)}\right|\right)
\end{aligned}
$$

We also have the von Neumann entropy of $\Lambda^{*}(\Phi)$ is

$$
S\left(\Lambda^{*}(\Phi)\right)=0=S(\Phi)
$$


The two marginal states of $\Lambda^{*}(\Phi)$ are

$$
\begin{aligned}
& \Lambda_{1}^{*} \rho_{1}=\frac{4}{9}\left|x_{0}^{(1)}\right\rangle\left\langle x_{0}^{(1)}\left|+\frac{4}{9}\right| x_{1}^{(1)}\right\rangle\left\langle x_{1}^{(1)}\left|+\frac{1}{9}\right| x_{2}^{(1)}\right\rangle\left\langle x_{2}^{(1)}\right| \\
& \Lambda_{2}^{*} \rho_{2}=\frac{4}{9}\left|x_{0}^{(2)}\right\rangle\left\langle x_{0}^{(2)}\left|+\frac{4}{9}\right| x_{1}^{(2)}\right\rangle\left\langle x_{1}^{(2)}\left|+\frac{1}{9}\right| x_{2}^{(2)}\right\rangle\left\langle x_{2}^{(2)}\right|
\end{aligned}
$$

The von Neumann entropy of two marginal states $\Lambda_{1}^{*} \rho_{1}$ and $\Lambda_{2}^{*} \rho_{2}$ are

$$
\begin{aligned}
& S\left(\Lambda_{1}^{*} \rho_{1}\right)=2 \log 3-\frac{16}{9} \log 2>S\left(\rho_{1}\right) \\
& S\left(\Lambda_{2}^{*} \rho_{2}\right)=2 \log 3-\frac{16}{9} \log 2>S\left(\rho_{2}\right)
\end{aligned}
$$

\section{Concluding discussion}

Here we present a new approach to the problem of order-stability in biosystems formulated by Schrödinger in 1944 [2]. This approach is based on the quantum-like paradigm realized in the framework of the open quantum systems theory. The following particular problem is studied: preservation of order-stability by biosystem $S$ compound of subsystems performing some biological functions generating disorder-increasing. In modelling we explored the features of quantum information processing, especially constancy of an isolated quantum information system entropy and the possibility to generate entangled states. The quantum-like model is purely informational, i.e., biosystems are considered as information processors; for each subsystem $S_{i}$, the rest of the compound system $S$ is treated as the information environment. The order-stability has the meaning of stability of information processing in $S$. Thus, this paper is a part of the information approach to physics and biology, from Wheeler's "It from bit" [5] to the recent information interpretation of quantum theory [6]-[12] and Johnson's emphasize that life without information processing is impossible [3]. Once again, we stress that this approach is not rigidly coupled to micro-world and it supports strongly the quantum-like paradigm - context sensitive systems, e.g., biosystems can process information in accordance with the laws of quantum information theory.

In this paper, we consider the simplest situation of an isolated compound biosystem $S$. The next step is modeling order stability of the quantum information state of a compound open system $S$ interacting with the information environment $\mathcal{E}_{S}$. Its state dynamics is non-unitary. In such a model, disorder in the biosystem $S$ is coming both from outside, namely from the information environment $\mathcal{E}_{S}$, and from inside, that is to say, the subsystems $S_{i}$ of $S$. Of course, the phenomenon of life is not reduced to order stability. But, even consistent modelling of information exchange stability in a complex biosystem is a step towards clarification of this phenomenon. The authors hope that this paper matches with Schrödinger vision [2] of information processes in biosystems (within modern quantum information representation).

\section{Appendix A: Unitarity of quantum channel: two dimensional state space}

Consider the case of the qubit state spaces of subsystems $S_{1}$ and $S_{2}$ of the compound system $S$ and the quantum channel from section 5.1. We present the proof of its unitarity:

Lemma 1. $V^{*} V=V V^{*}=I_{1} \otimes I_{2}$ 


\section{Proof.}

$$
\begin{aligned}
V^{*}= & \sqrt{\frac{1}{2}}\left|x_{0}^{(1)}\right\rangle \otimes\left|x_{0}^{(2)}\right\rangle\left(\left\langle x_{0}^{(1)}\right| \otimes\left\langle x_{0}^{(2)}\right|+\left\langle x_{1}^{(1)}\right| \otimes\left\langle x_{1}^{(2)}\right|\right) \\
& +\sqrt{\frac{1}{2}}\left|x_{1}^{(1)}\right\rangle \otimes\left|x_{1}^{(2)}\right\rangle\left(\left\langle x_{0}^{(1)}\right| \otimes\left\langle x_{0}^{(2)}\right|-\left\langle x_{1}^{(1)}\right| \otimes\left\langle x_{1}^{(2)}\right|\right) \\
& +\sqrt{\frac{1}{2}}\left|x_{0}^{(1)}\right\rangle \otimes\left|x_{1}^{(2)}\right\rangle\left(\left\langle x_{0}^{(1)}\right| \otimes\left\langle x_{1}^{(2)}\right|+\left\langle x_{1}^{(1)}\right| \otimes\left\langle x_{0}^{(2)}\right|\right) \\
& +\sqrt{\frac{1}{2}}\left|x_{1}^{(1)}\right\rangle \otimes\left|x_{0}^{(2)}\right\rangle\left(\left\langle x_{0}^{(1)}\right| \otimes\left\langle x_{1}^{(2)}\right|-\left\langle x_{1}^{(1)}\right| \otimes\left\langle x_{0}^{(2)}\right|\right)
\end{aligned}
$$

Then one has

$$
\begin{aligned}
V^{*} V= & \left(\left|x_{0}^{(1)}\right\rangle \otimes\left|x_{0}^{(2)}\right\rangle\right)\left(\left\langle x_{0}^{(1)}\right| \otimes\left\langle x_{0}^{(2)}\right|\right) \\
& +\left(\left|x_{1}^{(1)}\right\rangle \otimes\left|x_{1}^{(2)}\right\rangle\right)\left(\left\langle x_{1}^{(1)}\right| \otimes\left\langle x_{1}^{(2)}\right|\right) \\
& +\left(\left|x_{0}^{(1)}\right\rangle \otimes\left|x_{1}^{(2)}\right\rangle\right)\left(\left\langle x_{0}^{(1)}\right| \otimes\left\langle x_{1}^{(2)}\right|\right) \\
& +\left(\left|x_{1}^{(1)}\right\rangle \otimes\left|x_{0}^{(2)}\right\rangle\right)\left(\left\langle x_{1}^{(1)}\right| \otimes\left\langle x_{0}^{(2)}\right|\right) \\
= & \left|x_{0}^{(1)}\right\rangle\left\langle x_{0}^{(1)}|\otimes| x_{0}^{(2)}\right\rangle\left\langle x_{0}^{(2)}\right| \\
& +\left|x_{1}^{(1)}\right\rangle\left\langle x_{1}^{(1)}|\otimes| x_{1}^{(2)}\right\rangle\left\langle x_{1}^{(2)}\right| \\
& +\left|x_{0}^{(1)}\right\rangle\left\langle x_{0}^{(1)}|\otimes| x_{1}^{(2)}\right\rangle\left\langle x_{1}^{(2)}\right| \\
& +\left|x_{1}^{(1)}\right\rangle\left\langle x_{1}^{(1)}|\otimes| x_{0}^{(2)}\right\rangle\left\langle x_{0}^{(2)}\right| \\
= & \left|x_{0}^{(1)}\right\rangle\left\langle x_{0}^{(1)}\right| \otimes\left(\left|x_{0}^{(2)}\right\rangle\left\langle x_{0}^{(2)}|+| x_{1}^{(2)}\right\rangle\left\langle x_{1}^{(2)}\right|\right) \\
& +\left|x_{1}^{(1)}\right\rangle\left\langle x_{1}^{(1)}\right| \otimes\left(\left|x_{0}^{(2)}\right\rangle\left\langle x_{0}^{(2)}|+| x_{1}^{(2)}\right\rangle\left\langle x_{1}^{(2)}\right|\right) \\
= & \left|x_{0}^{(1)}\right\rangle\left\langle x_{0}^{(1)}\left|\otimes I_{2}+\right| x_{1}^{(1)}\right\rangle\left\langle x_{1}^{(1)}\right| \otimes I_{2} \\
= & \left.\left.|| x_{0}^{(1)}\right\rangle\left\langle x_{0}^{(1)}|+| x_{1}^{(1)}\right\rangle\left\langle x_{1}^{(1)}\right|\right) \otimes I_{2} \\
= & I_{2} \otimes I_{2}
\end{aligned}
$$


and

$$
\begin{aligned}
& V V^{*} \\
= & \frac{1}{2}\left(\left|x_{0}^{(1)}\right\rangle \otimes\left|x_{0}^{(2)}\right\rangle+\left|x_{1}^{(1)}\right\rangle \otimes\left|x_{1}^{(2)}\right\rangle\right)\left(\left\langle x_{0}^{(1)}\right| \otimes\left\langle x_{0}^{(2)}\right|+\left\langle x_{1}^{(1)}\right| \otimes\left\langle x_{1}^{(2)}\right|\right) \\
& +\frac{1}{2}\left(\left|x_{0}^{(1)}\right\rangle \otimes\left|x_{0}^{(2)}\right\rangle-\left|x_{1}^{(1)}\right\rangle \otimes\left|x_{1}^{(2)}\right\rangle\right)\left(\left\langle x_{0}^{(1)}\right| \otimes\left\langle x_{0}^{(2)}\right|-\left\langle x_{1}^{(1)}\right| \otimes\left\langle x_{1}^{(2)}\right|\right) \\
& +\frac{1}{2}\left(\left|x_{0}^{(1)}\right\rangle \otimes\left|x_{1}^{(2)}\right\rangle+\left|x_{1}^{(1)}\right\rangle \otimes\left|x_{0}^{(2)}\right\rangle\right)\left(\left\langle x_{0}^{(1)}\right| \otimes\left\langle x_{1}^{(2)}\right|+\left\langle x_{1}^{(1)}\right| \otimes\left\langle x_{0}^{(2)}\right|\right) \\
& +\frac{1}{2}\left(\left|x_{0}^{(1)}\right\rangle \otimes\left|x_{1}^{(2)}\right\rangle-\left|x_{1}^{(1)}\right\rangle \otimes\left|x_{0}^{(2)}\right\rangle\right)\left(\left\langle x_{0}^{(1)}\right| \otimes\left\langle x_{1}^{(2)}\right|-\left\langle x_{1}^{(1)}\right| \otimes\left\langle x_{0}^{(2)}\right|\right) \\
= & \frac{1}{2}\left(\left|x_{0}^{(1)}\right\rangle\left\langle x_{0}^{(1)}|\otimes| x_{0}^{(2)}\right\rangle\left\langle x_{0}^{(2)}|+| x_{0}^{(1)}\right\rangle\left\langle x_{1}^{(1)}|\otimes| x_{0}^{(2)}\right\rangle\left\langle x_{1}^{(2)}\right|\right. \\
& \left.+\left|x_{1}^{(1)}\right\rangle\left\langle x_{0}^{(1)}|\otimes| x_{1}^{(2)}\right\rangle\left\langle x_{0}^{(2)}|+| x_{1}^{(1)}\right\rangle\left\langle x_{1}^{(1)}|\otimes| x_{1}^{(2)}\right\rangle\left\langle x_{1}^{(2)}\right|\right) \\
& +\frac{1}{2}\left(\left|x_{0}^{(1)}\right\rangle\left\langle x_{0}^{(1)}|\otimes| x_{0}^{(2)}\right\rangle\left\langle x_{0}^{(2)}|-| x_{0}^{(1)}\right\rangle\left\langle x_{1}^{(1)}|\otimes| x_{0}^{(2)}\right\rangle\left\langle x_{1}^{(2)}\right|\right. \\
& \left.-\left|x_{1}^{(1)}\right\rangle\left\langle x_{0}^{(1)}|\otimes| x_{1}^{(2)}\right\rangle\left\langle x_{0}^{(2)}|+| x_{1}^{(1)}\right\rangle\left\langle x_{1}^{(1)}|\otimes| x_{1}^{(2)}\right\rangle\left\langle x_{1}^{(2)}\right|\right) \\
& +\frac{1}{2}\left(\left|x_{0}^{(1)}\right\rangle\left\langle x_{0}^{(1)}|\otimes| x_{1}^{(2)}\right\rangle\left\langle x_{1}^{(2)}|+| x_{0}^{(1)}\right\rangle\left\langle x_{1}^{(1)}|\otimes| x_{1}^{(2)}\right\rangle\left\langle x_{0}^{(2)}\right|\right. \\
& \left.+\left|x_{1}^{(1)}\right\rangle\left\langle x_{0}^{(1)}|\otimes| x_{0}^{(2)}\right\rangle\left\langle x_{1}^{(2)}|+| x_{1}^{(1)}\right\rangle\left\langle x_{1}^{(1)}|\otimes| x_{0}^{(2)}\right\rangle\left\langle x_{0}^{(2)}\right|\right) \\
& +\frac{1}{2}\left(\left|x_{0}^{(1)}\right\rangle\left\langle x_{0}^{(1)}|\otimes| x_{1}^{(2)}\right\rangle\left\langle x_{1}^{(2)}|-| x_{0}^{(1)}\right\rangle\left\langle x_{1}^{(1)}|\otimes| x_{1}^{(2)}\right\rangle\left\langle x_{0}^{(2)}\right|\right. \\
& \left.-\left|x_{1}^{(1)}\right\rangle\left\langle x_{0}^{(1)}|\otimes| x_{0}^{(2)}\right\rangle\left\langle x_{1}^{(2)}|+| x_{1}^{(1)}\right\rangle\left\langle x_{1}^{(1)}|\otimes| x_{0}^{(2)}\right\rangle\left\langle x_{0}^{(2)}\right|\right) \\
= & \left|x_{0}^{(1)}\right\rangle\left\langle x_{0}^{(1)}|\otimes| x_{0}^{(2)}\right\rangle\left\langle x_{0}^{(2)}|+| x_{1}^{(1)}\right\rangle\left\langle x_{1}^{(1)}|\otimes| x_{1}^{(2)}\right\rangle\left\langle x_{1}^{(2)}\right| \\
& +\left|x_{0}^{(1)}\right\rangle\left\langle x_{0}^{(1)}|\otimes| x_{1}^{(2)}\right\rangle\left\langle x_{1}^{(2)}|+| x_{1}^{(1)}\right\rangle\left\langle x_{1}^{(1)}|\otimes| x_{0}^{(2)}\right\rangle\left\langle x_{0}^{(2)}\right| \\
= & \left|x_{0}^{(1)}\right\rangle\left\langle x_{0}^{(1)}\right| \otimes\left(\left|x_{1}^{(2)}\right\rangle\left\langle x_{1}^{(2)}|+| x_{0}^{(2)}\right\rangle\left\langle x_{0}^{(2)}\right|\right) \\
& +\left|x_{1}^{(1)}\right\rangle\left\langle x_{1}^{(1)}\right| \otimes\left(\left|x_{1}^{(2)}\right\rangle\left\langle x_{1}^{(2)}|+| x_{0}^{(2)}\right\rangle\left\langle x_{0}^{(2)}\right|\right) \\
= & \left|x_{0}^{(1)}\right\rangle\left\langle x_{0}^{(1)}\left|\otimes I_{2}+\right| x_{1}^{(1)}\right\rangle\left\langle x_{1}^{(1)}\right| \otimes I_{2} \\
= & \left.\left.|| x_{0}^{(1)}\right\rangle\left\langle x_{0}^{(1)}|+| x_{1}^{(1)}\right\rangle\left\langle x_{1}^{(1)}\right|\right) \otimes I_{2} \\
& I_{1} \otimes I_{2}
\end{aligned}
$$

\section{Appendix B: Unitarity of the quantum channel: $N$-dimensional state space}

Here we present the proof of unitarity of the quantum channel from section 5.2, i.e., for the $N$-dimensional state spaces of two subsystems $S_{1}$ and $S_{2}$ of the compound system $S$.

Lemma 2. $V^{*} V=V V^{*}=I_{1} \otimes I_{2}$

\section{Proof.}

$$
V^{*}=\sum_{k, \ell=0}^{N-1}\left|x_{k}^{(1)}\right\rangle \otimes\left|x_{\ell}^{(2)}\right\rangle\left\langle\varphi_{k, \ell}\right|
$$




$$
\begin{aligned}
& V^{*}=\frac{2}{N}\left|x_{0}^{(1)}\right\rangle \otimes\left|x_{0}^{(2)}\right\rangle\left(\left(-\frac{N-2}{2}\right)\left\langle x_{0}^{(1)}\right| \otimes\left\langle x_{0}^{(2)}\right|+\left\langle x_{1}^{(1)}\right| \otimes\left\langle x_{1}^{(2)}\right|+\cdots+\left\langle x_{N-1}^{(1)}\right| \otimes\left\langle x_{N-1}^{(2)}\right|\right) \\
& +\frac{2}{N}\left|x_{0}^{(1)}\right\rangle \otimes\left|x_{1}^{(2)}\right\rangle\left(\left\langle x_{0}^{(1)}\right| \otimes\left\langle x_{0}^{(2)}\right|+\left(-\frac{N-2}{2}\right)\left\langle x_{1}^{(1)}\right| \otimes\left\langle x_{1}^{(2)}\right|+\cdots+\left\langle x_{N-1}^{(1)}\right| \otimes\left\langle x_{N-1}^{(2)}\right|\right) \\
& +\cdots+ \\
& +\frac{2}{N}\left|x_{0}^{(1)}\right\rangle \otimes\left|x_{N-1}^{(2)}\right\rangle\left(\left\langle x_{0}^{(1)}\right| \otimes\left\langle x_{0}^{(2)}\right|+\left\langle x_{1}^{(1)}\right| \otimes\left\langle x_{1}^{(2)}\right|+\cdots+\left(-\frac{N-2}{2}\right)\left\langle x_{N-1}^{(1)}\right| \otimes\left\langle x_{N-1}^{(2)}\right|\right) \\
& +\frac{2}{N}\left|x_{1}^{(1)}\right\rangle \otimes\left|x_{0}^{(2)}\right\rangle\left(\left(-\frac{N-2}{2}\right)\left\langle x_{0}^{(1)}\right| \otimes\left\langle x_{1}^{(2)}\right|+\left\langle x_{1}^{(1)}\right| \otimes\left\langle x_{2}^{(2)}\right|+\cdots+\left\langle x_{N-1}^{(1)}\right| \otimes\left\langle x_{0}^{(2)}\right|\right) \\
& +\frac{2}{N}\left|x_{1}^{(1)}\right\rangle \otimes\left|x_{1}^{(2)}\right\rangle\left(\left\langle x_{0}^{(1)}\right| \otimes\left(-\frac{N-2}{2}\right)\left\langle x_{1}^{(2)}\right|+\left\langle x_{1}^{(1)}\right| \otimes\left\langle x_{2}^{(2)}\right|+\cdots+\left\langle x_{N-1}^{(1)}\right| \otimes\left\langle x_{0}^{(2)}\right|\right) \\
& +\cdots+ \\
& +\frac{2}{N}\left|x_{1}^{(1)}\right\rangle \otimes\left|x_{N-1}^{(2)}\right\rangle\left(\left\langle x_{0}^{(1)}\right| \otimes\left\langle x_{1}^{(2)}\right|+\left\langle x_{1}^{(1)}\right| \otimes\left\langle x_{2}^{(2)}\right|+\cdots+\left(-\frac{N-2}{2}\right)\left\langle x_{N-1}^{(1)}\right| \otimes\left\langle x_{0}^{(2)}\right|\right) \\
& +\frac{2}{N}\left|x_{2}^{(1)}\right\rangle \otimes\left|x_{0}^{(2)}\right\rangle\left(\left(-\frac{N-2}{2}\right)\left\langle x_{0}^{(1)}\right| \otimes\left\langle x_{2}^{(2)}\right|+\left\langle x_{1}^{(1)}\right| \otimes\left\langle x_{3}^{(2)}\right|+\cdots+\left\langle x_{N-1}^{(1)}\right| \otimes\left\langle x_{1}^{(2)}\right|\right) \\
& +\frac{2}{N}\left|x_{2}^{(1)}\right\rangle \otimes\left|x_{1}^{(2)}\right\rangle\left(\left\langle x_{0}^{(1)}\right| \otimes\left\langle x_{2}^{(2)}\right|+\left(-\frac{N-2}{2}\right)\left\langle x_{1}^{(1)}\right| \otimes\left\langle x_{3}^{(2)}\right|+\cdots+\left\langle x_{N-1}^{(1)}\right| \otimes\left\langle x_{1}^{(2)}\right|\right) \\
& +\cdots+ \\
& +\frac{2}{N}\left|x_{2}^{(1)}\right\rangle \otimes\left|x_{N-1}^{(2)}\right\rangle\left(\left\langle x_{0}^{(1)}\right| \otimes\left\langle x_{2}^{(2)}\right|+\left\langle x_{1}^{(1)}\right| \otimes\left\langle x_{3}^{(2)}\right|+\cdots+\left(-\frac{N-2}{2}\right)\left\langle x_{N-1}^{(1)}\right| \otimes\left\langle x_{1}^{(2)}\right|\right) \\
& +\cdots \cdots+ \\
& +\frac{2}{N}\left|x_{N-1}^{(1)}\right\rangle \otimes\left|x_{0}^{(2)}\right\rangle\left(\left(-\frac{N-2}{2}\right)\left\langle x_{0}^{(1)}\right| \otimes\left\langle x_{N-1}^{(2)}\right|+\left\langle x_{1}^{(1)}\right| \otimes\left\langle x_{0}^{(2)}\right|+\cdots+\left\langle x_{N-1}^{(1)}\right| \otimes\left\langle x_{N-2}^{(2)}\right|\right) \\
& +\frac{2}{N}\left|x_{N-1}^{(1)}\right\rangle \otimes\left|x_{1}^{(2)}\right\rangle\left(\left\langle x_{0}^{(1)}\right| \otimes\left\langle x_{N-1}^{(2)}\right|+\left(-\frac{N-2}{2}\right)\left\langle x_{1}^{(1)}\right| \otimes\left\langle x_{0}^{(2)}\right|+\cdots+\left\langle x_{N-1}^{(1)}\right| \otimes\left\langle x_{N-2}^{(2)}\right|\right) \\
& +\cdots+ \\
& +\frac{2}{N}\left|x_{N-1}^{(1)}\right\rangle \otimes\left|x_{N-1}^{(2)}\right\rangle\left(\left\langle x_{0}^{(1)}\right| \otimes\left\langle x_{N-1}^{(2)}\right|+\left\langle x_{1}^{(1)}\right| \otimes\left\langle x_{0}^{(2)}\right|+\cdots+\left(-\frac{N-2}{2}\right)\left\langle x_{N-1}^{(1)}\right| \otimes\left\langle x_{N-2}^{(2)}\right|\right) \\
& V^{*} V=\sum_{k, \ell=0}^{N-1} \sum_{k^{\prime}, \ell^{\prime}=0}^{N-1}\left|x_{k}^{(1)}\right\rangle \otimes\left|x_{\ell}^{(2)}\right\rangle\left\langle\varphi_{k, \ell}|| \varphi_{k^{\prime}, \ell^{\prime}}\right\rangle\left\langle x_{k^{\prime}}^{(1)}\right| \otimes\left\langle x_{\ell^{\prime}}^{2}\right|
\end{aligned}
$$




$$
\begin{aligned}
& \left\langle\varphi_{k, \ell}, \varphi_{k^{\prime}, \ell^{\prime}}\right\rangle \\
& =\frac{4}{N^{2}} \sum_{j=0}^{N-1} \overline{\alpha_{k, \ell, j}}\left\langle x_{j}^{(1)}\left|\otimes\left\langle x_{j+k \bmod N}^{(2)}\left|\sum_{j^{\prime}=0}^{N-1} \alpha_{k^{\prime}, \ell^{\prime}, j^{\prime}}\right| x_{j^{\prime}}^{(1)}\right\rangle \otimes\right| x_{j^{\prime}+k^{\prime} \bmod \mathrm{N}}^{(2)}\right\rangle \\
& =\delta_{k . k^{\prime}} \delta_{\ell, \ell^{\prime}} \frac{4}{N^{2}} \delta_{j . j^{\prime}} \sum_{j=0}^{N-1} \sum_{j^{\prime}=0}^{N-1} \overline{\alpha_{k, \ell, j}} \alpha_{k^{\prime}, \ell^{\prime}, j^{\prime}}\left\langle x_{j}^{(1)}, x_{j^{\prime}}^{(1)}\right\rangle\left\langle x_{j+k \bmod N^{\prime}}^{(2)} x_{j^{\prime}+k^{\prime} \bmod \mathrm{N}}^{(2)}\right\rangle \\
& =\delta_{k \cdot k^{\prime}} \delta_{\ell, \ell^{\prime}} \frac{4}{N^{2}} \sum_{j=0}^{N-1}\left|\alpha_{k, \ell, j}\right|^{2} \\
& =\delta_{k \cdot k^{\prime}} \delta_{\ell, \ell^{\prime}} \frac{4}{N^{2}}\left(\left(-\frac{N-2}{2}\right)^{2}+(N-1)\right) \\
& =\delta_{k \cdot k^{\prime}} \delta_{\ell, \ell^{\prime}} \frac{4}{N^{2}}\left(\frac{(N-2)^{2}}{4}+\frac{4(N-1)}{4}\right) \\
& =\delta_{k . k^{\prime}} \delta_{\ell, \ell^{\prime}} \frac{4}{N^{2}}\left(\frac{N^{2}-4 N+4}{4}+\frac{4 N-4}{4}\right) \\
& =\delta_{k . k^{\prime}} \delta_{\ell, \ell^{\prime}} \frac{4}{N^{2}} \frac{N^{2}}{4} \\
& =\delta_{k \cdot k^{\prime}} \delta_{\ell, \ell^{\prime}} 1 \\
& V^{*} V=\sum_{k, \ell=0}^{N-1} \sum_{k^{\prime}, \ell^{\prime}=0}^{N-1}\left|x_{k}^{(1)}\right\rangle \otimes\left|x_{\ell}^{(2)}\right\rangle\left\langle\varphi_{k, \ell}|| \varphi_{k^{\prime}, \ell^{\prime}}\right\rangle\left\langle x_{k^{\prime}}^{(1)}\right| \otimes\left\langle x_{\ell^{\prime}}^{(2)}\right| \\
& =\sum_{k, \ell=0}^{N-1}\left|x_{k}^{(1)}\right\rangle \otimes\left|x_{\ell}^{(2)}\right\rangle\left\langle x_{k}^{(1)}\right| \otimes\left\langle x_{\ell}^{(2)}\right| \\
& =\sum_{k=0}^{N-1}\left|x_{k}^{(1)}\right\rangle\left\langle x_{k}^{(1)}\left|\otimes \sum_{\ell=0}^{N-1}\right| x_{\ell}^{(2)}\right\rangle\left\langle x_{\ell}^{(2)}\right| \\
& =I_{1} \otimes I_{2} \\
& V V^{*}=\sum_{k^{\prime}, \ell^{\prime}=0}^{N-1} \sum_{k, \ell=0}^{N-1}\left|\varphi_{k^{\prime}, \ell^{\prime}}\right\rangle\left\langle x_{k^{\prime}}^{(1)}\left|\otimes\left\langle x_{\ell^{\prime}}^{(2)}|| x_{k}^{(1)}\right\rangle \otimes\right| x_{\ell}^{(2)}\right\rangle\left\langle\varphi_{k, \ell}\right| \\
& =\sum_{k, \ell=0}^{N-1}\left|\varphi_{k, \ell}\right\rangle\left\langle\varphi_{k, \ell}\right|
\end{aligned}
$$




$$
\begin{aligned}
& \sum_{\ell=0}^{N-1}\left|\varphi_{k, \ell}\right\rangle\left\langle\varphi_{k, \ell}\right| \\
& =\sum_{\ell=0}^{N-1} \frac{4}{N^{2}} \sum_{j^{\prime}=0}^{N-1} \alpha_{k^{\prime}, \ell^{\prime}, j^{\prime}}\left|x_{j^{\prime}}^{(1)}\right\rangle \otimes\left|x_{j^{\prime}+k^{\prime} \bmod \mathrm{N}}^{(2)}\right\rangle \\
& \sum_{j=0}^{N-1} \overline{\alpha_{k, \ell, j}}\left\langle x_{j}^{(1)}\right| \otimes\left\langle x_{j+k \bmod \mathrm{N}}^{(2)}\right| \\
& =\sum_{\ell=0}^{N-1} \frac{4}{N^{2}} \sum_{j^{\prime}=0}^{N-1} \alpha_{k^{\prime}, \ell^{\prime}, j^{\prime}} \sum_{j=0}^{N-1} \overline{\alpha_{k, \ell, j}}\left|x_{j^{\prime}}^{(1)}\right\rangle\left\langle x_{j}^{(1)}|\otimes| x_{j^{\prime}+k^{\prime} \bmod \mathrm{N}}^{(2)}\right\rangle\left\langle x_{j+k \bmod \mathrm{N}}^{(2)}\right| \\
& =\sum_{\ell=0}^{N-1} \frac{4}{N^{2}} \sum_{j=0}^{N-1}\left|\alpha_{k, \ell, j}\right|^{2}\left|x_{j}^{(1)}\right\rangle\left\langle x_{j}^{(1)}|\otimes| x_{j+k \bmod \mathrm{N}}^{(2)}\right\rangle\left\langle x_{j+k \bmod \mathrm{N}}^{(2)}\right| \\
& +\frac{4}{N^{2}} 2\left(-\frac{N-2}{2}\right) \sum_{\ell=0 \wedge \ell \neq j}^{N-1} \sum_{j=0}^{N-1}\left|x_{\ell}^{(1)}\right\rangle\left\langle x_{j}^{(1)}|\otimes| x_{\ell+k \bmod \mathrm{N}}^{(2)}\right\rangle\left\langle x_{j+k \bmod \mathrm{N}}^{(2)}\right| \\
& +\frac{4}{N^{2}}(N-2) \sum_{\ell=0 \wedge \ell \neq j}^{N-1} \sum_{j=0}^{N-1}\left|x_{\ell}^{(1)}\right\rangle\left\langle x_{j}^{(1)}|\otimes| x_{\ell+k \bmod N}^{(2)}\right\rangle\left\langle x_{j+k \bmod \mathrm{N}}^{(2)}\right| \\
& =\frac{4}{N^{2}}(N-2) \sum_{j=0 \wedge j \neq \ell}^{N-1}\left|x_{j}^{(1)}\right\rangle\left\langle x_{j}^{(1)}|\otimes| x_{j+k \bmod \mathrm{N}}^{(2)}\right\rangle\left\langle x_{j+k \bmod \mathrm{N}}^{(2)}\right| \\
& +\sum_{\ell=0}^{N-1} \frac{(N-2)^{2}}{4}\left|x_{\ell}^{(1)}\right\rangle\left\langle x_{\ell}^{(1)}|\otimes| x_{\ell+k \bmod \mathrm{N}}^{(2)}\right\rangle\left\langle x_{\ell+k \bmod \mathrm{N}}^{(2)}\right| \\
& +\frac{4}{N^{2}}(-(N-2)+(N-2)) \sum_{\ell=0 \wedge \ell \neq j}^{N-1} \sum_{j=0}^{N-1}\left|x_{\ell}^{(1)}\right\rangle\left\langle x_{j}^{(1)}|\otimes| x_{\ell+k \bmod \mathrm{N}}^{(2)}\right\rangle\left\langle x_{j+k \bmod \mathrm{N}}^{(2)}\right| \\
& =\sum_{\ell=0}^{N-1} \frac{4}{N^{2}} \frac{N^{2}}{4}\left|x_{\ell}^{(1)}\right\rangle\left\langle x_{\ell}^{(1)}|\otimes| x_{\ell+k \bmod \mathrm{N}}^{(2)}\right\rangle\left\langle x_{\ell+k \bmod \mathrm{N}}^{(2)}\right| \\
& =\sum_{\ell=0}^{N-1}\left|x_{\ell}^{(1)}\right\rangle\left\langle x_{\ell}^{(1)}|\otimes| x_{\ell+k \bmod \mathrm{N}}^{(2)}\right\rangle\left\langle x_{\ell+k \bmod \mathrm{N}}^{(2)}\right|
\end{aligned}
$$




$$
\begin{aligned}
& V V^{*} \\
& =\frac{4}{N^{2}}\left(\left(-\frac{N-2}{2}\right)\left|x_{0}^{(1)}\right\rangle \otimes\left|x_{0}^{(2)}\right\rangle+\left|x_{1}^{(1)}\right\rangle \otimes\left|x_{1}^{(2)}\right\rangle+\cdots+\left|x_{N-1}^{(1)}\right\rangle \otimes\left|x_{N-1}^{(2)}\right\rangle\right) \\
& \left(\left(-\frac{N-2}{2}\right)\left\langle x_{0}^{(1)}\right| \otimes\left\langle x_{0}^{(2)}\right|+\left\langle x_{1}^{(1)}\right| \otimes\left\langle x_{1}^{(2)}\right|+\cdots+\left\langle x_{N-1}^{(1)}\right| \otimes\left\langle x_{N-1}^{(2)}\right|\right) \\
& +\frac{4}{N^{2}}\left(\left|x_{0}^{(1)}\right\rangle \otimes\left|x_{0}^{(2)}\right\rangle+\left(-\frac{N-2}{2}\right)\left|x_{1}^{(1)}\right\rangle \otimes\left|x_{1}^{(2)}\right\rangle+\cdots+\left|x_{N-1}^{(1)}\right\rangle \otimes\left|x_{N-1}^{(2)}\right\rangle\right) \\
& \left(\left\langle x_{0}^{(1)}\right| \otimes\left\langle x_{0}^{(2)}\right|+\left(-\frac{N-2}{2}\right)\left\langle x_{1}^{(1)}\right| \otimes\left\langle x_{1}^{(2)}\right|+\cdots+\left\langle x_{N-1}^{(1)}\right| \otimes\left\langle x_{N-1}^{(2)}\right|\right) \\
& +\cdots+ \\
& +\frac{4}{N^{2}}\left(\left|x_{0}^{(1)}\right\rangle \otimes\left|x_{0}^{(2)}\right\rangle+\left|x_{1}^{(1)}\right\rangle \otimes\left|x_{1}^{(2)}\right\rangle+\cdots+\left(-\frac{N-2}{2}\right)\left|x_{N-1}^{(1)}\right\rangle \otimes\left|x_{N-1}^{(2)}\right\rangle\right) \\
& \left(\left\langle x_{0}^{(1)}\right| \otimes\left\langle x_{0}^{(2)}\right|+\left\langle x_{1}^{(1)}\right| \otimes\left\langle x_{1}^{(2)}\right|+\cdots+\left(-\frac{N-2}{2}\right)\left\langle x_{N-1}^{(1)}\right| \otimes\left\langle x_{N-1}^{(2)}\right|\right) \\
& +\frac{4}{N^{2}}\left(\left(-\frac{N-2}{2}\right)\left|x_{0}^{(1)}\right\rangle \otimes\left|x_{1}^{(2)}\right\rangle+\left|x_{1}^{(1)}\right\rangle \otimes\left|x_{2}^{(2)}\right\rangle+\cdots+\left|x_{N-1}^{(1)}\right\rangle \otimes\left|x_{0}^{(2)}\right\rangle\right) \\
& \left(\left(-\frac{N-2}{2}\right)\left\langle x_{0}^{(1)}\right| \otimes\left\langle x_{1}^{(2)}\right|+\left\langle x_{1}^{(1)}\right| \otimes\left\langle x_{2}^{(2)}\right|+\cdots+\left\langle x_{N-1}^{(1)}\right| \otimes\left\langle x_{0}^{(2)}\right|\right) \\
& +\cdots+ \\
& +\frac{4}{N^{2}}\left(\left|x_{0}^{(1)}\right\rangle \otimes\left|x_{1}^{(2)}\right\rangle+\left|x_{1}^{(1)}\right\rangle \otimes\left|x_{2}^{(2)}\right\rangle+\cdots+\left(-\frac{N-2}{2}\right)\left|x_{N-1}^{(1)}\right\rangle \otimes\left|x_{0}^{(2)}\right\rangle\right) \\
& \left(\left\langle x_{0}^{(1)}\right| \otimes\left\langle x_{1}^{(2)}\right|+\left\langle x_{1}^{(1)}\right| \otimes\left\langle x_{2}^{(2)}\right|+\cdots+\left(-\frac{N-2}{2}\right)\left\langle x_{N-1}^{(1)}\right| \otimes\left\langle x_{0}^{(2)}\right|\right) \\
& +\cdots+ \\
& +\frac{4}{N^{2}}\left(\left(-\frac{N-2}{2}\right)\left|x_{0}^{(1)}\right\rangle \otimes\left|x_{N-1}^{(2)}\right\rangle+\left|x_{1}^{(1)}\right\rangle \otimes\left|x_{0}^{(2)}\right\rangle+\cdots+\left|x_{N-1}^{(1)}\right\rangle \otimes\left|x_{N-2}^{(2)}\right\rangle\right) \\
& \left(\left(-\frac{N-2}{2}\right)\left\langle x_{0}^{(1)}\right| \otimes\left\langle x_{N-1}^{(2)}\right|+\left\langle x_{1}^{(1)}\right| \otimes\left\langle x_{0}^{(2)}\right|+\cdots+\left\langle x_{N-1}^{(1)}\right| \otimes\left\langle x_{N-2}^{(2)}\right|\right) \\
& +\cdots+ \\
& +\frac{4}{N^{2}}\left(\left|x_{0}^{(1)}\right\rangle \otimes\left|x_{N-1}^{(2)}\right\rangle+\left|x_{1}^{(1)}\right\rangle \otimes\left|x_{0}^{(2)}\right\rangle+\cdots+\left(-\frac{N-2}{2}\right)\left|x_{N-1}^{(1)}\right\rangle \otimes\left|x_{N-2}^{(2)}\right\rangle\right) \\
& \left(\left\langle x_{0}^{(1)}\right| \otimes\left\langle x_{N-1}^{(2)}\right|+\left\langle x_{1}^{(1)}\right| \otimes\left\langle x_{0}^{(2)}\right|+\cdots+\left(-\frac{N-2}{2}\right)\left\langle x_{N-1}^{(1)}\right| \otimes\left\langle x_{N-2}^{(2)}\right|\right)
\end{aligned}
$$

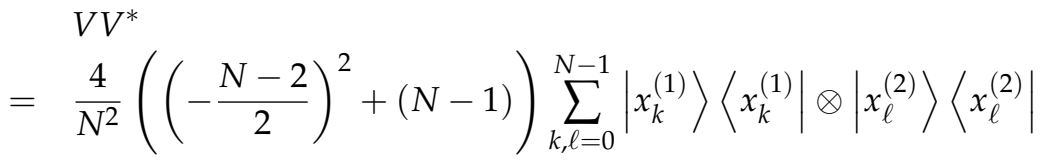

$$
\begin{aligned}
& +\frac{4}{N^{2}}\left(2\left(-\frac{N-2}{2}\right)+(N-2)\right) \sum_{k \neq k^{\prime}=0}^{N-1} \sum_{\ell \neq \ell^{\prime}=0}^{N-1}\left|x_{k}^{(1)}\right\rangle\left\langle x_{k^{\prime}}^{(1)}|\otimes| x_{\ell}^{(2)}\right\rangle\left\langle x_{\ell^{\prime}}^{(2)}\right| \\
& =\sum_{k=0}^{N-1}\left|x_{k}^{(1)}\right\rangle\left\langle x_{k}^{(1)}\left|\otimes \sum_{\ell=0}^{N-1}\right| x_{\ell}^{(2)}\right\rangle\left\langle x_{\ell}^{(2)}\right| \\
& =I_{1} \otimes I_{2}
\end{aligned}
$$




$$
\begin{aligned}
V V^{*} & =\sum_{k, \ell=0}^{N-1}\left|\varphi_{k, \ell}\right\rangle\left\langle\varphi_{k, \ell}\right| \\
& =\sum_{k, \ell=0}^{N-1}\left|x_{\ell}^{(1)}\right\rangle\left\langle x_{\ell}^{(1)}|\otimes| x_{\ell+k \bmod \mathrm{N}}^{(2)}\right\rangle\left\langle x_{\ell+k \bmod \mathrm{N}}^{(2)}\right| \\
& =\sum_{\ell=0}^{N-1}\left|x_{\ell}^{(1)}\right\rangle\left\langle x_{\ell}^{(1)}\left|\otimes \sum_{k=0}^{N-1}\right| x_{k}^{(2)}\right\rangle\left\langle x_{k}^{(2)}\right| \\
& =I_{1} \otimes I_{2}
\end{aligned}
$$

Author Contributions: Formal analysis, N.W.; Methodology, A.K.; Calculations: N.W.; Paper writing: A.K. and N.W,; All authors have read and agreed to the published version of the manuscript.

Funding: This research received no external funding.

Conflicts of Interest: The authors declare no conflict of interest.

\section{References}

1. Khrennikov, A. Information Dynamics in Cognitive, Psychological, Social, and Anomalous Phenomena; Springer: Dordrecht, Netherlands, 2004.

2. Schrödinger, E. What is Life?; Cambridge Univ. Press: Cambridge, 1944.

3. Johnson, H. A. Information theory in biology after 18 years. Science 1970, 168, 1545-1550.

4. Gatenby, R. A. \& Frieden, R. Information theory in living systems, methods, applications, and challenges. Bull. Math. Biology 2007, 69(2), 635-57.

5. Wheeler, J. A. . Information, physics, quantum: The search for links. In: Zurek, W. H. (ed.) Complexity, Entropy, and the Physics of Information. Addison-Wesley, Redwood City, 1990, 354-368.

6. Fuchs, C. A. Quantum mechanics as quantum information (and only a little more), in A. Khrennikov (ed.), Quantum Theory: Reconsideration of Foundations, Ser. Math. Model., 2 (Växjö University Press, Växjö), 2002, pp. 463-543.

7. Caves, C. M.; Fuchs, C. A. \& Schack, R. Quantum probabilities as Bayesian probabilities, Phys. Rev. A, 2002, 65, 022305.

8. Plotnitsky, A. Epistemology and Probability: Bohr, Heisenberg, Schrödinger and the Nature of Quantum-Theoretical Thinking; Springer: Berlin, Germany; New York, NY, USA, 2009.

9. Brukner, C. \& Zeilinger, A. Information invariance and quantum probabilities, Found. Phys. 2009, 39, 677-688.

10. Chiribella, G.; D'Ariano, G. M. \& Perinotti, P. Probabilistic theories with purification, Phys. Rev. A 2020, 81, 062348.

11. Chiribella, G.; D'Ariano, G. M. \& Perinotti, P. Informational axioms for quantum theory. In: Foundations of Probability and Physics - 6, AIP Conf. Proc. 1424, 2012, pp. 270-279.

12. Fuchs,C. A. \& Khrennikov, A. Preface to special issue. Quantum information revolution: Impact to foundations. Found. Phys. 2020, 50, 1757-1761.

13. Ingarden, R. S.; Kossakowski, A. \& Ohya, M. Information Dynamics and Open Systems: Classical and quantum approach; Kluwer: Dordrecht, 1997.

14. Asano, M.; Khrennikov, A.; Ohya, M.; Tanaka, Y. \& Yamato, I. Quantum Adaptivity in Biology: from Genetics to Cognition; Springer: Heidelberg-Berlin-New York, 2015.

15. Penrose, R. The Emperor's New Mind; Oxford Univ. Press: New-York, 1989.

16. Umezawa, H. Advanced Field Theory: Micro, Macro and Thermal Concepts; AIP: New York, 1993.

17. Hameroff, S. Quantum coherence in microtubules. A neural basis for emergent consciousness? J. Cons. Stud. 1994, 1, 91-118.

18. Vitiello, G. Dissipation and memory capacity in the quantum brain model. Int. J. Mod. Phys. 1995, B9, 973.

19. Vitiello, G. My Double Unveiled: The Dissipative Quantum Model of Brain; Advances in Consciousness Research, John Benjamins Publishing Company, 2001. 
20. Khrennikov, A. Classical and quantum mechanics on information spaces with applications to cognitive, psychological, social and anomalous phenomena, Found. Physics 1999, 29, 1065-1098.

21. Khrennikov, A. Quantum-like formalism for cognitive measurements. Biosystems 2003, 70, 211-233.

22. Khrennikov, A. On quantum-like probabilistic structure of mental information, Open Systems and Information Dynamics 2004, 11 (3), 267-275.

23. Khrennikov, A. Information Dynamics in Cognitive, Psychological, Social, and Anomalous Phenomena, Ser: Fundamental Theories of Physics; Kluwer: Dordreht, 2004.

24. Khrennikov, A. Ubiquitous Quantum Structure: from Psychology to Finances; Springer Berlin-Heidelberg-New York, 2010.

25. Busemeyer, J. \& Bruza, P. Quantum Models of Cognition and Decision; Cambridge Univ. Press: Cambridge, 2012.

26. Haven, E. Pilot-wave theory and financial option pricing, Int. J. Theor. Phys. 2005, 44 (11), 1957-1962.

27. Khrennikov, A. Quantum-like brain: Interference of minds, BioSystems 2006, 84, 225-241.

28. Busemeyer, J. R.; Wang, Z. \& Townsend, J. T. Quantum dynamics of human decision making, J. Math. Psych. 2006, 50, 220-241.

29. Pothos, E. \& Busemeyer J. R. A quantum probability explanation for violations of 'rational' decision theory, Proceedings of Royal Society B 2009, 276, 2171-2178.

30. Yukalov, V.I. \& Sornette, D. Physics of risk and uncertainty in quantum decision making. Eur. Phys. J. B 2009, $71,533-548$.

31. Asano, M. Ohya, M.; Tanaka, Y.; Basieva, I. \& Khrennikov, A. Quantum-like model of brain's functioning: decision making from decoherence. J. Theor. Biology 2011, 281(1), 56-64.

32. Dzhafarov, E. N. \& Kujala, J. V. (2012). Selectivity in probabilistic causality: Where psychology runs into quantum physics, J. Math. Psych., 56, 54-63.

33. Asano, M.; Basieva, I.; Khrennikov, A,; Ohya, M.; Tanaka, Y, \& Yamato, I. Quantum-like model for the adaptive dynamics of the genetic regulation of E. coli's metabolism of glucose-lactose. Syst Synth Biol 2012, $6,1-7$.

34. Asano, M.; Basieva, I.; Khrennikov, A,; Ohya, M.; Tanaka, Y, \& Yamato, I. Towards modeling of epigenetic evolution with the aid of theory of open quantum Systems. AIP Conference Proceedings 1508, 75 (2012); https:/ / doi.org/10.1063/1.4773118

35. Bagarello, F. \& Oliveri, F. A phenomenological operator description of interactions between populations with applications to migration, Mathematical Models and Methods in Applied Sciences 2013, 23 (03), 471-492.

36. Wang, Z. \& Busemeyer, J. R. A quantum question order model supported by empirical tests of an a priori and precise prediction. Topics in Cognitive Science 2013, 5, 689-710.

37. Wang, Z.; Solloway, T.; Shiffrin, R. M. \& Busemeyer, J. R. Context effects produced by question orders reveal quantum nature of human judgments. PNAS 2014, 111, 9431-9436.

38. Khrennikov, A. \& Basieva, I. Quantum model for psychological measurements: from the projection postulate to interference of mental observables represented as positive operator valued measures. NeuroQuantology 2014, 12, 324-336.

39. Yukalov, V.I.\& Sornette, D. Conditions for quantum interference in cognitive sciences Top. Cogn. Science 2014, 6, 79 - 90 .

40. Khrennikov, A. \& Basieva, I. Possibility to agree on disagree from quantum information and decision making, J. Math. Psychology 2014, 62(3), 1-5.

41. Khrennikova, P. A quantum framework for 'Sour Grapes' in cognitive dissonance. In: Atmanspacher H., Haven E., Kitto K., Raine D. (eds) Quantum Interaction. QI 2013. Lecture Notes in Computer Science 2014, 8369. Springer, Berlin, Heidelberg.

42. Busemeyer, J. R.; Wang, Z.; Khrennikov, A. \& Basieva, I. Applying quantum principles to psychology Physica Scripta 2014, T163, 014007.

43. Asano, M.; Khrennikov, A.; Ohya, M.; Tanaka, Y.\& Yamato, I. Violation of contextual generalization of the Leggett-Garg inequality for recognition of ambiguous figures. Physica Scripta 2014, T163, 014006.

44. Khrennikov, A.; Basieva, I.; Dzhafarov, E.N. \& Busemeyer, J. R. Quantum models for psychological measurements: An unsolved problem. PLOS ONE 2014, 9, Art. e110909.

45. Boyer-Kassem, T.; Duchene, S. Guerci, E. Quantum-like models cannot account for the conjunction fallacy, Theor. Decis. 2015, 10, 1-32. 
46. Dzhafarov, E. N.; Zhang, R. \& Kujala, J. V. Is there contextuality in behavioral and social systems? Phil. Trans. Royal Soc. A 2015, 374, 20150099.

47. Asano, M.; Basieva, I.; Khrennikov, A.; Ohya, M.; Tanaka, Y. \& Yamato, I. Quantum information biology: from information interpretation of quantum mechanics to applications in molecular biology and cognitive psychology. Found. Phys. 2015, 45, 1362-1378.

48. Basieva, I. \& Khrennikov, A. On the possibility to combine the order effect with sequential reproducibility for quantum measurements. Found. Physics 2015, 45(10), 1379-1393.

49. Khrennikov, A. Quantum-like model of unconscious-conscious dynamics; Frontiers in Psychology 2015, 6, Art. N 997.

50. Khrennikov, A. Quantum Bayesianism as the basis of general theory of decision-making. Phil. Trans. R. Soc. A 2016, 374, 20150245.

51. Khrennikova, P. Quantum dynamical modeling of competition and cooperation between political parties: the coalition and non-coalition equilibrium model. J. Math. Psych. 2016, 71, 39-50.

52. Asano, M.; Basieva, I.; Khrennikov, A. \& Yamato, I. A model of differentiation in quantum bioinformatics. Progress in Biophysics and Molecular Biology Part A 2017, 130, 88-98.

53. Yukalov, V.I.\& Sornette, D. Quantum probabilities as behavioral probabilities. Entropy 2017, 19, 112.

54. Asano, M.; Basieva, I.; Khrennikov, A.; Ohya, M. \& Tanaka, Y., A quantum-like model of selection behavior. J. Math. Psych. 2017, 78, 2-12.

55. Khrennikova, P. Modeling behavior of decision makers with the aid of algebra of qubit creation-annihilation operators, J. Math. Psych. 2017, 78, 76-85.

56. Basieva, I.; Pothos, E.; Trueblood, J.; Khrennikov, A. \& Busemeyer, J. Quantum probability updating from zero prior (by-passing Cromwell's rule), J. Math. Psych. 2017, 77, 58-69.

57. Khrennikov, A.; Basieva, I.; Pothos, E. M. \& Yamato, I. Quantum probability in decision making from quantum information representation of neuronal states, Scientific Reports 2018, 8, 16225.

58. Lawless, W. F. The interdependence of autonomous human-machine teams: The entropy of teams, but not individuals, Advances science. Entropy 2019, 21(12), 1195.

59. Bagarello, F.; Gargano, F. \& Oliveri, F. Spreading of competing information in a network. Entropy 2020, 22(10), 1169.

60. Ozawa, M. \& Khrennikov, A. Application of theory of quantum instruments to psychology: Combination of question order effect with response replicability effect. Entropy, 2020 22(1), 37.

61. Yukalov, V. I. Evolutionary processes in quantum decision theory. Entropy 2020, 22(6), 68.

62. Kolmolgoroff, A. N. Grundbegriffe der Wahrscheinlichkeitsrechnung; Springer-Verlag: Berlin, 1933.

63. Khrennikov, A. Contextual Approach to Quantum Formalism; Springer: Berlin, Germany; New York, NY, USA, 2009.

64. Khrennikov, A. Probability and Randomness: Quantum Versus Classical; Imperial College Press: London, 2016.

65. Khrennikov, A. \& Alodjants, A. Classical (local and contextual) probability model for Bohm-Bell type experiments: No-Signaling as independence of random variables. Entropy 2019, 21, 157-177.

66. Chernega, V.N., Man'ko, O.V. \& Man'ko, V.I. Correlations in a system of classical-like coins simulating spin-1/2 states in the probability representation of quantum mechanics. Eur. Phys. J. D 2019, 73, 10.

67. Chernega, V.N., Man'ko, O.V. \& Man'ko, V.I. Probability representation of quantum states as a renaissance of hidden variables — God plays coins. J. Russian Laser Research 2019, 40, 107-120. 